\title{
Association of Body Mass Index with Left Ventricular Diastolic Dysfunction Among Ambulatory Patients with Diabetes Mellitus in Rural Uganda: A Cross Sectional Study.
}

Boniface Amanee Elias Lumori ( $\sim$ lboniface@must.ac.ug )

Mbarara University of science and Technology Mbarara

\section{Edwin Nuwagira}

Mbarara University of science and Technology Mbarara

Fardous Charles Abeya

Mbarara University of science and Technology Mbarara

Abdirahman Ali Araye

Mbarara University of science and Technology Mbarara

Godfrey Masete

Mbarara University of science and Technology Mbarara

Charles K. Mondo

Makerere University

\section{Samson Okello}

Mbarara University of science and Technology Mbarara

\section{Conrad Muzoora}

Mbarara University of science and Technology Mbarara

Anthony Muyingo

Mbarara University of science and Technology Mbarara

\section{Research Article}

Keywords: Left ventricular diastolic dysfunction, Diabetes mellitus, Ambulatory patients, Body mass index, rural Uganda

Posted Date: December 10th, 2021

DOl: https://doi.org/10.21203/rs.3.rs-1140954/v1

License: (c) (i) This work is licensed under a Creative Commons Attribution 4.0 International License. Read Full License 


\section{Abstract}

Background: Left ventricular diastolic dysfunction (LVDD) is a recognized complication of diabetes mellitus which precedes and is a risk factor for heart failure. We aimed to determine the prevalence of LVDD and its association with body mass index (BMI) in ambulatory adults with diabetes mellitus.

Methods: We conducted a cross-sectional study of 195 ambulatory Ugandan adults living with diabetes mellitus for at least five years from the time of diagnosis at Mbarara Regional Referral Hospital (MRRH). We collected demographic, clinical data and measured body mass index. Laboratory tests included glycated hemoglobin ( $\mathrm{HbA1c}$ ), low-density lipoprotein (LDL), and urine microalbumin. Echocardiography was done to determine LVDD by assessing the mitral inflow E/A ratio, E/è ratio, TR jet peak velocity, and left atrium maximum volume index. Logistic regression was used to establish associations of body mass index and other covariates with LVDD.

Results: Overall, 195 participants were enrolled and 141(72.3\%) were females. The mean age was 62 [standard deviation (SD), 11.5] years and median duration of diabetes diagnosis was 10 [Interquartile range (IQR), 7,15] years. LVDD was prevalent at $86 \%$ and majority, $127(65.13 \%)$ had grade-1 diastolic dysfunction. $\mathrm{BMI} \geq 25 \mathrm{~kg} / \mathrm{m}^{2}$ [adjusted Odds Ratio $(\mathrm{aOR})=2.8$, (95\% confidence interval $\left.(\mathrm{Cl})=1-7.6\right)$, $\mathrm{p}=0.038]$, age 50 year or more [aOR=4.9, $(95 \% \mathrm{Cl}=1.5-16.2), \mathrm{p}=0.010]$, and history of hypertension [aOR= 3.0, $(95 \% \mathrm{Cl}=1.1-8.1), \mathrm{p}=0.031]$ were associated with LVDD.

Conclusion: The prevalence of LVDD was high during the study period. We recommend early and periodic echocardiographic screening for diabetes patients with high body mass index.

\section{Background}

Globally, four hundred million adults are living with diabetes mellitus and Africa carries a huge burden accounting for 25 million[1, 2]. The prevalence of diabetes mellitus in Uganda recorded among urban and rural populations were at $7.6 \%$ and $16.1 \%$, respectively[3]. Despite advances in preventive and therapeutic care, individuals with diabetes have up to eight folds risk of cardiovascular disease(CVD)[4] and congestive heart failure is high as $23 \%$, which is three times higher compared to non-diabetic controls[5]. More than two-thirds of all mortalities in diabetic patients are due to CVD[4, 6]. Even more, in Africa, the mortality is high and often goes undiagnosed[7, 8]. Aside from atherosclerotic and coronary diseases, several pathways including LVDD play a key role early in the pathogenesis of heart failure and contribute to this mortality burden $[4,9,10]$. LVDD itself is associated with increased risk of subclinical atherosclerosis and ischemic heart disease[11]. In diabetes, the etiology of LVDD is often multifactorial with chronic hyperglycemia, overweight/obesity, and hypertension as the main pathogenic factors that eventually lead to morphological and physiological changes of the heart muscle[9] [12]. A survey in rural Uganda showed high prevalence of these cardiovascular risk factors among diabetic patients with prehypertension/hypertension prevalent in $70 \%$; and overweight /obesity at $21 \%$ of the studied population[13]. These factors synergistically worsen and predict diastolic dysfunction, ischemic heart 
diseases, and subsequently overt heart failure[9, 14]. Increased BMI is modifiable risk factor that could be a potential cheap interventional target to reduce the prevalence of LVDD in diabetic patients.

Although echocardiographic screening of diabetic patients enables early detection of LVDD before overt heart failure, in low resource countries like Uganda, a routine echocardiography is not being done which represents a missed opportunity for optimization of treatment and screening[15]. In this study, we aimed to determine the prevalence of LVDD and its association with body mass index on ambulatory adults with diabetes mellitus attending an outpatient clinic in rural Uganda.

\section{Methods}

\section{Study design and setting}

This was a cross-sectional study of adult patients with diabetes mellitus presenting for routine care in the diabetes clinic of MRRH. Mbarara Regional Referral Hospital is the largest referral hospital in southwestern Uganda. It is a public university teaching hospital for both postgraduate and undergraduate students of Mbarara University of Science and Technology (MUST). The hospital is sponsored by the government of Uganda through the Ministry of Health $(\mathrm{MOH})$. It is approximately $260 \mathrm{~km}$ southwestern of Kampala, the capital city.

The diabetes clinic currently serves more than 1,500 patients. The average patients' attendance per each clinic day is 70, and about two-third get seen by clinicians and the rest come for refilling of medications. The clinic is run by a senior internal medicine physician with vast experience in diabetes care and management, at least two internal medicine postgraduate students, one medical officer, and two certified nurses. The clinic nurses register patients, record vital signs, do fasting (or random) blood sugar test, educate patients on medications adherence, and explain to the patients the importance of appointment dates.

\section{Recruitment and eligibility criteria}

Patients were identified by a research assistant during their routine registration in the clinic book and then screened consecutively using the study eligibility criteria. We included all adults of at least 18 years with history of diabetes for at least 5 years. We excluded patients with documented history of heart failure, valvular heart disease, arrhythmia, thyroid disorders, and those with history of hazardous intake of alcohol (based on Alcohol Use Disorder Identification Test) as well as patients with poor echocardiographic window as these conditions modify left ventricular structure and/or function. Participants who declined consent were further excluded.

\section{Data collection procedure and variables}

We used a standardized quantitative questionnaire to collect data from history, physical findings, laboratory, and Echocardiographic parameters. The history included age, occupational status, educational level, duration of diabetes, hypertension and smoking habit. We then measured the blood 
pressure and calculated $\mathrm{BMI}$ from weight and height. The blood pressure was measured in the supine position using OMRAN HEM-8712 automatic blood pressure monitor (Omran Corporation, Kyoto, Japan) twice 30 minutes apart and only the second blood pressure measurement was considered. We used ADE automatic weighing scale and portable stadiometer (Hamburg, Germany) for the weights and heights, respectively. This was followed by removal of $4-5 \mathrm{mls}$ of blood for HbA1c and LDL tests. The SiemensDCA Vantage ${ }^{T M}$ analyzer (Siemens Healthcare Diagnostics Ltd, Frimley, Camberley, UK) was used to analyze the HbA1c while HumaStar 200 Automated Analyzer (Human Diagnostics Uganda) for LDL. Five to ten mls of urine was collected for a spot urine albumin to creatinine ratio (UACR) test which was analyzed using Combina 13 Test Strips (Human Diagnostics Uganda). All samples were analyzed at Mbarara University clinic research laboratory which has standardized internal quality control protocols and participates in external quality control programs by the National Health Laboratory Service. Furthermore, a trained internist performed echocardiography (Philips HD7 XE Diagnostic ultrasound system, China) according to the recommendations of American Society of Echocardiography and the European Association of cardiovascular imaging 2016 guidelines for the evaluation of left ventricular diastolic function. The LVDD was classified as grade 1 if a mitral inflow E/A ratio $\leq 0.8$ with peak $E$ velocity $\leq 50 \mathrm{~cm} / \mathrm{sec}$ [or $E / A \leq 0.8$ and peak $E>50 \mathrm{~cm} / \mathrm{sec}$ or $E / A>0.8$ but $<2$ plus either TR jet peak velocity $>2.8 \mathrm{~m} / \mathrm{sec}$, average $E / \mathrm{è}>14$ (or lateral $E /$ è ratio $>13$ or septal $E / \mathrm{è}>15$ ) or LA maximum volume index $>34 \mathrm{ml} / \mathrm{m}^{2}$ ]; grade 2 if E/A ratio $>0.8$ and $<2$ (or $\leq 0.8$ and peak E velocity is $>50 \mathrm{~cm} / \mathrm{sec}$ ) with at

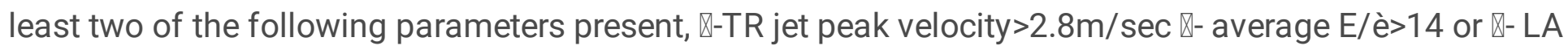
maximum volume index $>34 \mathrm{ml} / \mathrm{m}^{2}$; and grade 3 if E/A ratio $\geq 2$ [16].

\section{Statistical analysis}

All data were entered into Excel 2013 (Microsoft Corporation, Redmond, USA) spreadsheet and imported into the STATA 13 (College Station, Texas, USA) in which analysis was performed. The normally distributed continuous variables were summarized by means and the skewed continuous variables by medians. Categorical variables were grouped by frequencies and percentages. The prevalence of LVDD was determined by a simple proportion command. Univariate and multivariate logistic regression were used to establish associations of $\mathrm{BMI}$ and other biological plausible variables with LVDD. A $P$ value of $\leq 0.05$ was considered to indicate a statistically significant association.

\section{Ethics}

This study was reviewed and approved by the Department of Internal Medicine, Faculty of Medicine Research Committee, and the Research Ethics Committee of Mbarara University of Science and Technology. Informed consent was obtained from all respondents and confidentiality was maintained throughout the study.

\section{The study participants' recruitment}

In the period from November 2017 to March 2018, we screened 998 patients with diabetes mellitus. Of these, 782 had diabetes mellitus of less than five years' duration, five had documentation of atrial 
fibrillation, four had hazardous intake of alcohol, and one with history of hyperthyroidism. In addition, nine declined informed consent and two had poor echocardiographic window (figure 1).

\section{Results}

The mean age was 62 (SD, 11.5) years and 141(72.3\%) were females. The median duration of diabetes was $10($ IQR 7,15$)$ years. Most of the participants had a primary level of education, $91(46.67 \%)$ and majority were peasant farmers, $108(55.38 \%)$. One hundred and forty-six $(74.87 \%)$ had history of hypertension and most never smoked, 160 (82.05\%). Overall, 125(64.10\%) had symptoms of burry vision and $119(61.03 \%)$ had paresthesia at enrollment. Most participants had high BMI, 70(36.08\%) were overweight (BMI, $25-29.9 \mathrm{~kg} / \mathrm{m}^{2}$ ) and $60(30.93 \%)$ were obese (BMI, $\geq 30 \mathrm{~kg} / \mathrm{m}^{2}$ ). The median blood pressure measured at enrollment was $145(128,158)$ for systolic blood pressure (SBP) and $81(75,89)$ for diastolic pressure. The medians (IQR) for HbA1c, LDL, and UACR were 9.1(7.7,10.9), 3.02(2.3,3.9), and 266.6(100,500), respectively (Table 1$)$. 
Table 1

Baseline socio-demographic and clinical characteristics

\begin{tabular}{|c|c|}
\hline Characteristic & $\mathrm{N}=195$ \\
\hline Age in years, mean ( $\left.{ }^{\star} S D\right)$ & $62(11.5)$ \\
\hline Female gender, $\mathrm{n}(\%)$ & $141(72.31)$ \\
\hline \multicolumn{2}{|l|}{ Level of education, $\mathrm{n}(\%)$ : } \\
\hline None & $47(24.10)$ \\
\hline Primary & $91(46.67)$ \\
\hline Secondary & $31(15.90)$ \\
\hline Tertiary & $26(13.33)$ \\
\hline \multicolumn{2}{|l|}{ Occupation, n (\%): } \\
\hline Peasant farming & $108(55.38)$ \\
\hline Business & 19(9.74) \\
\hline Employed & $30(15.38)$ \\
\hline Others & $38(19.47)$ \\
\hline Duration of diabetes in years, median ( $\left.{ }^{\star} \mid \mathrm{QR}\right)$ & $10(7,15)$ \\
\hline History of hypertension, $\mathrm{n}(\%)$ & $146(74.87)$ \\
\hline \multicolumn{2}{|l|}{ History of smoking, $\mathrm{n}(\%)$ : } \\
\hline Never & $160(82.05)$ \\
\hline Current & $1(0.51)$ \\
\hline Former & $34(17.44)$ \\
\hline \multicolumn{2}{|l|}{ Symptoms at enrolment, $\mathrm{n}(\%)$ : } \\
\hline Paraesthesia & $119(61.03)$ \\
\hline Blurry vision & $125(64.10)$ \\
\hline Erectile dysfunction & $15(7.69)$ \\
\hline Others & $49(64.10)$ \\
\hline Systolic blood pressure at enrolment in mmHg, median (IQR) & $145(128,158)$ \\
\hline Diastolic blood pressure at enrolment in mmHg, median (IQR) & $81(75,89)$ \\
\hline
\end{tabular}




\begin{tabular}{|ll|}
\hline Characteristic & $\mathrm{N}=195$ \\
\hline Underweight (below 18.5) & $5(2.58)$ \\
\hline Normal (18.5-24.9) & $59(30.41)$ \\
Overweight (25-29.9) & $70(36.08)$ \\
Obesity (30 and above) & $60(30.93)$ \\
\hline Positive *HIV status, $\mathrm{n}(\%)$ & $14(7.18)$ \\
\hline Glycated haemoglobin in \%, median (IQR) & $9.1(7.7,10.9)$ \\
\hline Glycated haemoglobin categories in \%, $\mathrm{n}(\%):$ & \\
\hline Less than 7 & $27(13.85)$ \\
\hline 7 and more & $168(86.15)$ \\
\hline Low density lipoproteins in mmol/L, median (IQR) & $3.02(2.3,3.9)$ \\
\hline Low density lipoproteins categories in mmol/L, $\mathrm{n}(\%):$ & \\
\hline Optimal (<2.6) & $71(36.41)$ \\
\hline Near optimal/above optimal (2.6-3.3) & $39(20.00)$ \\
\hline Borderline high (3.4-4.1) & $50(25.64)$ \\
\hline High (4.2-4.9) & $18(9.23)$ \\
Very high ( $\geq 5)$ & $17(8.72)$ \\
\hline Urine albumin creatinine ratio in mg/mol, median (IQR) & $266.6(100,500)$ \\
\hline Left ventricular diastolic function categories, $\mathrm{n}(\%):$ & $127(65.13)$ \\
\hline Normal & $31(15.90)$ \\
\hline Grade 1 diastolic dysfunction & $10(5.13)$ \\
\hline Grade 2 diastolic dysfunction & \\
Grade 3 diastolic dysfunction & \\
\hline
\end{tabular}

The prevalence of LVDD was found at 86. 15\% [std. error=0.025 (95\% Cl=0.8,0.9)]. Overall, $127(65.13 \%)$ had grade 1 diastolic dysfunction (Table 1$)$. We found that $\mathrm{BMI} \geq 25 \mathrm{~kg} / \mathrm{m}^{2}[\mathrm{aOR}=2.8(95 \% \mathrm{Cl}=(1,7.6)$, $p=0.038$ ], age of 50 years or more [aOR $=4.9(95 \% \mathrm{Cl}=1.5,16.2), p=0.010]$, and history of hypertension $[\mathrm{aOR}=3(95 \% \mathrm{Cl}=(1.1,8.1), p=0.031]$ were associated with LVDD (Table 2$)$. 
Table 2

Factors associated with increased odds of left ventricular diastolic dysfunction, $n=195$

\begin{tabular}{|c|c|c|c|c|}
\hline Variable & $\begin{array}{l}\text { *uOR } \\
(95 \% * \mathrm{Cl})\end{array}$ & $\begin{array}{l}P- \\
\text { value }\end{array}$ & $\begin{array}{l}\text { *aOR } \\
(95 \% \mathrm{Cl})\end{array}$ & $\begin{array}{l}P \text { - } \\
\text { value }\end{array}$ \\
\hline Age $\geq 50$ years & $5(1.8,13.6)$ & 0.002 & $\begin{array}{l}4.9(1.5 \\
16.2)\end{array}$ & 0.010 \\
\hline Female gender & $1.1(0.4,2.7)$ & 0.809 & $1.4(0.4,3.8)$ & 0.546 \\
\hline Duration of diabetes (each year increase) & $1(0.9,1)$ & 0.416 & $1(0.9,1)$ & 0.694 \\
\hline History of hypertension & $4(1.7,9.4)$ & 0.001 & $3(1.1,8.1)$ & 0.031 \\
\hline History of *smoking & $1.3(0.4,4)$ & 0.648 & $1.3(0.3,5.8)$ & 0.705 \\
\hline Body mass index category $\geq 25 \mathrm{~kg} / \mathrm{m}^{2}$ & $2.5(1.1,5.7)$ & 0.027 & $2.8(1,7.6)$ & 0.038 \\
\hline Positive HIV status & $2.2(0.2,17.3)$ & 0.462 & $\begin{array}{l}2.1(0.2 \\
21.4)\end{array}$ & 0.508 \\
\hline Glycated hemoglobin (each \% increase) & $1(0.8,1.1)$ & 0.853 & $1(0.8,1.2)$ & 0.988 \\
\hline Low density lipoproteins (each mmol/L increase) & $1(0.9,1)$ & 0.408 & $1(0.9,1)$ & 0.219 \\
\hline $\begin{array}{l}\text { Urine albumin to creatine ratio (each } \mathrm{mg} / \mathrm{mol} \\
\text { increase) }\end{array}$ & $1(0.9,1)$ & 0.480 & $1(0.9,1)$ & 0.516 \\
\hline
\end{tabular}

Table 2 legend: uOR: Unadjusted odds ratio, aOR; Adjusted odds ratio, Cl: Confidence Interval, smoking; current and former smokers

\section{Discussion}

In this study, the local prevalence of LVDD was very high among ambulatory adults with diabetes mellitus in rural Uganda and BMI $\geq 25 \mathrm{~kg} / \mathrm{m}^{2}$ was independently associated with LVDD. In addition, age $\geq 50$ years and having a history of hypertension were associated with increased odds of LVDD.

Our prevalence was generally high compared to prior studies done in Africa and other continents. In the study done in Mulago National Referral Hospital by Muddu and colleagues, the prevalence of LVDD was at 55\%[17] and in Egypt[18], the prevalence was at 61\%. Both studies recruited newly diagnosed patients with diabetes mellitus whereas our study focused on experienced diabetic patients, hence the higher prevalence in our study. Experienced diabetic patients have prolonged hyperglycemia, more hypertension, overweight/obesity, and dyslipidemia amongst other comorbid conditions which are known to cause LVDD[19-21]. In Nigeria, the prevalence of LVDD was 79\%[22], which is slightly lower compared to our study. The population in our study were more experienced than theirs. Older age has been shown in several studies to increase the risk of LVDD through increased left ventricular wall stiffness [23, 24]. 
Also, in Asian countries, lower prevalence compared to our study was recorded. In a study done in Iraq, the prevalence of LVDD was found at $62 \%[25]$ and in India, a prevalence of $54 \%$ was recorded[26]. Both studies had younger populations compared to those in our study and the latter, in addition, excluded hypertensive patients. Moreover, in Europe, a study done by Jorgensen and colleagues found a prevalence of $19 \%[27]$, which is much lower than what we found. This huge difference could probably be explained by the fact that the European study population was recruited from highly specialized centers of diabetic care with proper management, multidisciplinary care, and follow up, hence less diabetic related cardiac complications.

Similar to our study, previous studies recorded high BMI was associated with $\operatorname{LVDD}[18,27,28,33]$. Body mass index in our population might be a fairly stable factor compared to low density lipoprotein, the latter must be taken after an overnight fasting to produce a reliable result. Overweight and obesity is associated with increased plasma membrane content of fatty acids transporters which increase fatty acids uptake and utilization leading to cardiac steotosis and hence myocardial damage[12]. Nevertheless, Muddu et al found no association between high BMI and diabetic cardiomyopathy[17].

Prior studies in Africa, Asia, and Europe similarly found advanced age was associated with LVDD[14, 17, $25,27,28]$. This consistency could be explained by stiffening of the left ventricle wall which occurs with increase in age hence abnormal diastolic function[23, 24]. However, the Egyptian study did not record a similar result. This contradiction could partially be explained by their exclusion of patients older than 60 years and use of a complex echocardiographic technique by including certain tissue doppler imaging parameters[18].

Unsurprisingly, history of hypertension was found to be associated with LVDD in our study. Several studies have reported hypertension as one of the major causes of left ventricular hypertrophy and diastolic dysfunction[28-31]. Hypertension directly triggers remodeling of cardiac walls through increase in the afterloads and indirectly through the neurohormonal factors, particularly the renin-angiotensin system[32]. On contrary, for unknown reasons, Muddu and his colleagues didn't find association of hypertension with LVDD in their multivariate model[17].

Increased levels of $\mathrm{HbA} 1 \mathrm{c}$, microalbuminuria, and longer duration of diabetes were demonstrated in some studies as predictors of $\operatorname{LVDD}[14,18,27]$. However, this study and others[17, 34] didn't uncover a similar finding. This could partly be explained by lack of persistent glycemic control over time in our diabetic patients.

\section{Conclusion}

In this study, the prevalence of LVDD was high and high BMI is associated with increased odds of LVDD.

\section{Recommendations}


We recommend early and periodic echocardiographic screening for diabetic outpatients with high BMI. Future studies should focus on whether reduction of body mass index could reduce the prevalence of LVDD in diabetic adults.

\section{Abbreviations}

aOR: Adjusted odds ratio, BMI: Body Mass Index, Cl: Confidence Interval, CVD: Cardiovascular disease, HbA1c: glycated hemoglobin, IQR: Interquartile range, LDL: low density lipoproteins, LVDD: left ventricular diastolic dysfunction, MOH: Ministry of Health, MRRH: Mbarara Regional Referral Hospital, MUST: Mbarara University of Science and Technology, SBP: systolic blood pressure, UACR: urine albumin to creatinine ratio, uOR: Unadjusted odds ratio.

\section{Declarations}

\section{Ethics approval and consent to participate}

This study was reviewed and approved by the Department of Internal Medicine, Faculty of Medicine Research Committee (under the reference number: DMS 6), and the Research Ethics Committee of Mbarara University of Science and Technology (under the reference number: MUREC 1/7). We confirm that all eligible participants provided informed written consent to participate in this study. All methods were carried out in accordance with relevant guidelines and regulations.

\section{Consent for publication}

Not applicable.

\section{Availability of data and material}

The datasets used during this study are available from the corresponding

authors on reasonable request.

\section{Competing interests}

The authors have no conflict of interest to declare.

\section{Funding}

None.

\section{Authors' contributions}

BAEL-Conceptualized the study, edited protocol, collected data, analyze, interpreted data, wrote and edited the manuscript. 
EN- Edited protocol and contributed to the manuscript.

FAC-Edited protocol and contributed to the manuscript.

AAA-Edited protocol and contributed to the manuscript.

GM-Edited protocol and contributed to the manuscript.

CKM-Edited protocol and contributed to the manuscript.

So- Supervise, edited the protocol, and contributed to the manuscript

$\mathrm{CM}$ - Supervise, edited the protocol, and contributed to the manuscript.

AM-Supervise, edited the protocol, and contributed to the manuscript.

Co-authors SO, CM, and AM contributed equally to this research and manuscript.

All authors read and approved the final manuscript.

Acknowledgements

We acknowledge the study participants and all those who contributed to this work.

\section{References}

1. WHO, Global report on diabetes. 2016: World Health Organization.

2. ADA, Diagnosis and classification of diabetes mellitus. Diabetes care, 2014. 37(Supplement 1): p. S81S90.

3. Chiwanga, F.S., et al., Urban and rural prevalence of diabetes and pre-diabetes and risk factors associated with diabetes in Tanzania and Uganda. 2016. 9(1): p. 31440.

4. Martín-Timón, l., et al., Type 2 diabetes and cardiovascular disease: have all risk factors the same strength? World journal of diabetes, 2014. 5(4): p. 444.

5. Zhou, L., et al., Prevalence, incidence and risk factors of chronic heart failure in the type 2 diabetic population: systematic review. Current diabetes reviews, 2009. 5(3): p. 171-184.

6. Aitila, P., et al., Prevalence and risk factors of Helicobacter pylori infection among children aged 1 to 15 years at holy innocents children's hospital, Mbarara, South Western Uganda. 2019. 2019.

7. Aminde, L.N., A. Dzudie, and A.P.J.B.o. Kengne, Prevalent diabetes mellitus in patients with heart failure and disease determinants in sub-Saharan Africans having diabetes with heart failure: a protocol for a systematic review and meta-analysis. 2016. 6(2): p. e010097. 
8. Mbanya, J. and K. Ramiaya, Disease and Mortality in Sub-Saharan Africa, Diabetes Mellitus [Online] 2006 [access 2012, March 6]. 1752.

9. Battiprolu, P.K., et al., Diabetic cardiomyopathy: mechanisms and therapeutic targets. Drug Discovery Today: Disease Mechanisms, 2010. 7(2): p. e135-e143.

10. Kasznicki, J. and J.J.A.o.m.s.A. Drzewoski, Heart failure in the diabetic population-pathophysiology, diagnosis and management. 2014. 10(3): p. 546.

11. Jamiel, A., et al., Correlation between diastolic dysfunction and coronary artery disease on coronary computed tomography angiography. 2016. 17(1): p. 13.

12. Pappachan, J.M., et al., Diabetic cardiomyopathy: Pathophysiology, diagnostic evaluation and management. World journal of diabetes, 2013. 4(5): p. 177.

13. Namusisi, 0. , et al., Risk factors for non-communicable diseases in rural Uganda: a pilot surveillance project among diabetes patients at a referral hospital clinic. Pan African Medical Journal, 2011. 10.

14. Patil, V.C., et al., Diastolic dysfunction in asymptomatic type 2 diabetes mellitus with normal systolic function. Journal of cardiovascular disease research, 2011. 2(4): p. 213-222.

15. Voulgari, C., D. Papadogiannis, and N. Tentolouris, Diabetic cardiomyopathy: from the pathophysiology of the cardiac myocytes to current diagnosis and management strategies. Vascular health and risk management, 2010. 6(1): p. 883-903.

16. Nagueh, S.F., et al., Recommendations for the evaluation of left ventricular diastolic function by echocardiography: an update from the American Society of Echocardiography and the European Association of Cardiovascular Imaging. 2016. 17(12): p. 1321-1360.

17. Muddu, M., E. Mutebi, and C. Mondo, Prevalence, types and factors associated with echocardiographic abnormalities among newly diagnosed diabetic patients at Mulago Hospital. African health sciences, 2016. 16(1): p. 183-193.

18. Ayman, K.H., et al., Correlation between left ventricular diastolic dysfunction and dyslipidaemia in asymptomatic patients with new-onset type 2 diabetes mellitus. 2021. 33(1): p. 1-11.

19. Battiprolu, P.K., et al., Diabetic cardiomyopathy: mechanisms and therapeutic targets. 2010. 7(2): p. e135-e143.

20. Pappachan, J.M., et al., Diabetic cardiomyopathy: Pathophysiology, diagnostic evaluation and management. 2013. 4(5): p. 177.

21. Landsberg, L., M.J.C. Molitch, and e. hypertension, Diabetes and hypertension: pathogenesis, prevention and treatment. 2004. 26(7-8): p. 621-628. 
22. Dodiyi-Manuel, S.T., M.R. Akpa, and O.J. Odia, Left ventricular dysfunction in normotensive type II diabetic patients in Port Harcourt, Nigeria. Vascular health and risk management, 2013. 9: p. 529.

23. Salmasi, A.-M., et al., Age-associated changes in left ventricular diastolic function are related to increasing left ventricular mass. American journal of hypertension, 2003. 16(6): p. 473-477.

24. Pyszko, J., et al., Effects of age on left ventricular diastolic function. 2018.

25. Ashour, K.J.J.H.C.R., Early detection of diastolic dysfunction in diabetic patients (Single Center Cross Sectional Study). 2018. 2(1): p. 114.

26. Patil, V.C., et al., Diastolic dysfunction in asymptomatic type 2 diabetes mellitus with normal systolic function. 2011. 2(4): p. 213-222.

27. Jørgensen, P.G., et al., Abnormal echocardiography in patients with type 2 diabetes and relation to symptoms and clinical characteristics. Diabetes and Vascular Disease Research, 2016: p. 1479164116645583.

28. Srivastava, P.M., et al., Prevalence and predictors of cardiac hypertrophy and dysfunction in patients with Type 2 diabetes. 2008. 114(4): p. 313-320.

29. Palmiero, P., et al., Left ventricular diastolic function in hypertension: methodological considerations and clinical implications. 2015. 7(3): p. 137.

30. Ike, S. and V.J.G.m.j. Ikeh, The prevalence of diastolic dysfunction in adult hypertensive Nigerians. 2006. 40(2): p. 55.

31. Ratnaparkhe, V. and A.J.T.J.o.t.A.o.P.o.I. Bhangale, Left Ventricular Diastolic Dysfunction in Primary Hypertension and its Relation with Leisure Time Physical Activity. 2015. 63(7): p. 20-24.

32. Georgiopoulou, V.V., et al., Prevention, diagnosis, and treatment of hypertensive heart disease. Cardiology clinics, 2010. 28(4): p. 675-691.

33. Wai, B., et al., Prevalence, predictors and evolution of echocardiographically defined cardiac abnormalities in adults with type 1 diabetes: an observational cohort study. 2014. 28(1): p. 22-28.

34. Rothangpui, S.D.S., et al., Diabetic cardiomyopathy in Manipur. Indian journal of endocrinology and metabolism, 2011. 15(3): p. 204.

\section{Figures}

Figure 1 
Flow chart showing participants' recruitment.

Page 14/14 